\title{
MULTIGRAPH SPECTRAL CLUSTERING FOR JOINT CONTENT DELIVERY AND SCHEDULING IN BEAM-FREE SATELLITE COMMUNICATIONS
}

\author{
Miguel Ángel Vázquez $z^{\star}$ Ana I. Pérez-Neira ${ }^{\star \dagger}$ \\ * Centre Tecnològic de Telecomunicacions de Catalunya (CTTC/CERCA), Castelldefels (Spain). \\ $\dagger$ Dept. of Signal Theory and Communications, Universitat Politècnica de Catalunya, Barcelona (Spain).
}

\begin{abstract}
This paper tackles the problem of user scheduling in satellite content delivery networks with precoding. The clustering process has to consider two crucial and independent characteristics of the user terminals. On the one hand, users belonging to the same group shall have a reduced Euclidean norm between their channel vectors in order to obtain the maximum precoding gain. On the other hand, with the aim of exploiting the multicast capabilities of the system, user terminals grouped in the same cluster shall have requested the same content. The resulting clustering problem is formulated as a multigraph (also known as multiview) spectral clustering problem. The paper shows that this unsupervised learning framework is able to capture the different peculiarities of the mentioned problem. Two different techniques are introduced and validated in a close-to-real numerical simulation.
\end{abstract}

\section{INTRODUCTION}

In the early adoptions of $5 \mathrm{G}$, the satellite segment is identified as a key enabler of content distribution to the edge infrastructure [1]. The capability of reaching a large set of content requests in a single transmission results an attractive feature for content providers. Note that this is done by the intrinsic multicast transmission nature of the satellite system. In this paper, we investigate the problem of user scheduling in a content delivery satellite system with precoding. Although the recent success of precoding for increasing the satellite throughput is known [2], a study dealing with user scheduling in contentcentric systems is missing.

Scheduling in multibeam satellite systems employing precoding has been investigated in the recent years [2-9]. A scheduling technique that considers the rate fairness as a key performance indicator can be found in [3]. Most of all satellite standards embed more than one UT information in the same codeword in order to obtain high channel coding gains. Due to that, the scheduling process consists of grouping users from the same beam to be served over the same frame. Attending the UT channel vector, the work in [4] proposes to opportunistically group users based on their fed back signalto-interference-plus-noise ratio (SINR). The work in [5] clusters UTs which are geographically close. In [6] it is proposed to schedule UT having similar channel vectors over the

This work has received funding from the Spanish ministry of Science, Innovation and Universities under project TERESA-TEC2017-90093-C3-1-R (AEI/FEDER,UE), and by the Catalan Government under grant 2017-SGR01479 . same frame and schedule UTs in adjacent beams considering orthogonal channel vectors. A similar approach is done in [9] but only considering the geographical location of the UTs. In $[2,7,10]$ the authors consider user scheduling of UTs presenting the minimum Euclidean distance of their channel. This notion is extended in [8] by considering the $k$-means algorithm [11] and alternative similarity UT channel vector metrics.

None of the mentioned techniques consider the user grouping problem from the joint perspective of the precoding and content delivery operation as we perform in the paper. In particular, we aim to combine two crucial aspects of next generation satellite system. First, user scheduling in systems with precoding shall be driven by its channel vector characteristics. Second, in order to exploit the benefits of the multicast transmission, users requesting the same content shall be grouped together in order to minimize the communication overhead.

Bearing in mind the good performance of spectral clustering [12] for user grouping in beam-free satellite systems with precoding [13], in here we opt to consider the framework of multigraph clustering [14]. In multigraph (also known as multiview) clustering, the learning is performed over different undirected weighted graphs that share the same vertices but different weights. For our case, we assume that each UT is represented by a vertex and two different graphs are constructed; namely, one that informs about the channel vectors and another one is related to the content requests of each UT. Different techniques based on spectral clustering are analysed and validated in a real multibeam coverage area. To the best of authors knowledge, this is the first time multigraph spectral clustering is employed in the wireless communication arena. Based on the numerical results, this unsupervised learning approach is a promising solution for clustering wireless heterogeneous data aiming to support the next generation contentcentric radio resource management mechanisms.

\section{CONTENT DELIVERY AND PRECODING IN MULTIBEAM SATELLITE SYSTEMS}

\subsection{System Model}

We consider a forward link transmission of a single geostationary satellite system, consisting of one satellite payload equipped with an array fed reflector with $N$ feed elements and $K$ satellite user terminals (UTs). The UT set is denoted by $\mathcal{K}=\{1,2, \ldots, K\}$. Adhering to the commercial satellite system scenario, we focus on the case where $K>>N$. 
The forward link channels between the gateway $(\mathrm{GW})$ and the UTs are described by the channel matrix $\mathbf{H}=$ $\left(\mathbf{h}_{1}, \mathbf{h}_{2}, \ldots, \mathbf{h}_{K}\right)^{T} \in \mathbb{C}^{K \times N}$, where $\mathbf{h}_{k} \in \mathbb{C}^{N \times 1}$ denotes the channel between the gateway (GW) and the $k$-th UT. We adopt the line-of-sight channel model described in [2].

We assume that perfect channel state information is available at the GW. The GW performs user scheduling and serves the $K$ users in $B$ groups. The $B$ simultaneous data frames transport content from more than one UT, leading to a multigroup multicast transmission. We denote as $\mathcal{B}_{i}$ for $i=1, \ldots, B$ the set of groups. We assume that $\mathcal{B}_{i} \cap \mathcal{B}_{j}=\emptyset$ for $i \neq j$ and $\bigcup_{i=1, \ldots, B} \mathcal{B}_{i}=\mathcal{K}$.

Each UT is interested in a certain content (e.g. film, television show,...). The set of contents is denoted by $\mathcal{C}=1, \ldots, C$. Vectors $\mathbf{c}_{k} \in \mathbb{R}^{C \times 1}$ for $k=1, \ldots, K$ represent the $k$-th UT content requests: if the $i$-th content $(i=1, \ldots, C)$ is requested by the $k$-th UT, then $\left[\mathbf{c}_{k}\right]_{i}=1$ or 0 otherwise. In order to exploit the multicast capabilities of the satellite transmission, it is convenient that users requesting the same content are grouped over the same transmission.

In this preliminary study, we consider that over each data frame it is possible to encapsulate all content devoted to the cluster. Assuming a finite length frame error correction (FEC) formats involves solving a high complex problem where different FEC options shall be tested considering the content lengths. This study is beyond the scope of the current paper and it is left for further works.

It is important to remark that in this paper we consider a beam-free satellite architecture. In contrast to current systems where the satellite payload radiates fixed beams and each beam has its own dedicated network manager (i.e. scheduler), in here we assume an holistic scheduling entity able to group UTs from adjacent beams into the same transmission. This approach is aligned with future trends in high throughput satellites [13].

The $B$ simultaneous data streams are precoded using the minimum mean square error multicast (MMSE-M) technique [10]. While having significantly lower computational complexity than other approaches [6], the sum-rate performance of the MMSE-M is generally good for diverse multibeam satellite systems. We adopt per-feed power allocation to ensure that none of the satellite high power amplifiers reach the saturation. Then, the precoded signal is given by $\mathbf{x}=\mathbf{W} \mathbf{s}=\gamma\left(\mathbf{G}^{H} \mathbf{G}+\frac{B}{P} \mathbf{I}_{B}\right)^{-1} \mathbf{G}^{H} \mathbf{s}$, where matrix $\mathbf{W}=\left(\mathbf{w}_{1}, \mathbf{w}_{2}, \ldots, \mathbf{w}_{B}\right) \in \mathbb{C}^{N \times B}$ is the precoding matrix and $\mathbf{s} \in \mathbb{C}^{B \times 1}$ are the data symbols transmitted to the $B$ UTs groups. The data symbols are assumed to have unit power, i.e., $\mathbb{E}\left(\mathbf{s s}^{H}\right)=\mathbf{I}_{B}$. Matrix $\mathbf{G}=\left(\mathbf{g}_{1}, \mathbf{g}_{2}, \ldots, \mathbf{g}_{B}\right)^{T} \in \mathbb{C}^{B \times N}$ is constructed by $\mathbf{g}_{i}=\frac{1}{\left|\mathcal{B}_{i}\right|} \sum_{k \in \mathcal{B}_{i}} \mathbf{h}_{k}$, which is the centroid of the channel vectors of the UTs served in the same group. The scalar $\gamma$ is set such that the transmit power at each feed power amplifier is bellow $P: \gamma^{2}=P / \max _{n}\left[\mathbf{W} \mathbf{W}^{H}\right]_{n, n}$.

The overall receive signal model is $\mathbf{y}=\mathbf{H x}+\mathbf{n}$, where $\mathbf{n} \in \mathbb{C}^{K \times 1}$ is the additive white Gaussian noise with zero mean and unit variance. Hence, the signal-to-interferenceplus noise ratio (SINR) experience at each UT is $\mathrm{SINR}_{k}=$ $\frac{\left|\mathbf{h}_{k}^{H} \mathbf{w}_{k}\right|^{2}}{\sum_{j \neq k}^{B}\left|\mathbf{h}_{k}^{H} \mathbf{w}_{j}\right|^{2}+1}$.

\subsection{User Grouping Problem}

In general, typical satellite transmissions require that data from more than one UT is embedded into one frame. This means that the $\mathrm{GW}$ has to partition the set of users $(\mathcal{K})$ to be served into $B$ disjoint groups. Bearing in mind the user grouping considering both the precoding and the content delivery tasks, we propose to optimize the following weighted sum-rate problem

$$
\underset{\left\{\mathcal{B}_{i}\right\}_{i=1}^{B}}{\operatorname{maximize}} \sum_{i=1}^{B} \eta_{i} \min _{k \in \mathcal{B}_{i}} B_{W} \log _{2}\left(1+\operatorname{SINR}_{k}\right),
$$

where

$$
\eta_{i}=1-\frac{\sum_{m \in \mathcal{B}_{i}} \sum_{n>m, n \in \mathcal{B}_{i}}\left\|\mathbf{c}_{m}-\mathbf{c}_{n}\right\|^{2}}{C\left|\mathcal{B}_{i}\right|}
$$

Note that $\eta_{i}$ describes the multicast efficiency of the $i$-th cluster. Indeed, $\eta_{i}$ becomes 1 if all UTs belonging to the $i$-th group request the same content. Furthermore, it can be observed in the optimization problem in (1) that the attainable rate of each of the groups is given by the achievable rate of the user with the lowest SINR of that group. This is due to the multicast transmission: all UTs belonging to the same group have to be able to decode the transmitted frame.

Even though we fix the number of clusters, $B$, finding the optimal solution of the problem in (1) requires an exhaustive search over all possible $\mathcal{B}_{i}$. Let us clarify why clustering techniques are an adequate heuristic approach for solving the optimization problem in (1). First, if we only consider the maximization multicast efficiency such as

$$
\underset{\left\{\mathcal{B}_{i}\right\}_{i=1}^{B}}{\operatorname{maximize}} \sum_{i=1}^{B} \eta_{i}
$$

it is clear that the optimal solution is the one that provides a user grouping where each member of the group has the most similar content requests $\mathbf{c}_{k} \quad k=1, \ldots, K$. From the clustering perspective, this yields to user grouping that minimizes the intracluster distances [15]. Consequently, a clustering technique considering the content requests is an adequate approach for optimizing the problem in (3).

Second, if we consider the optimization problem with only the sum-rate

$$
\underset{\left\{\mathcal{B}_{i}\right\}_{i=1}^{B}}{\operatorname{maximize}} \sum_{i=1}^{B} \min _{k \in \mathcal{B}_{i}} B_{W} \log _{2}\left(1+\operatorname{SINR}_{k}\right),
$$

performing a user clustering considering the channel vectors $\mathbf{h}_{k} \quad k=1, \ldots, K$ and the Euclidean distance between them is a convenient heuristic approach. In particular, if spectral clustering is performed the operation approximately solves the partioning RatioCut problem [12]. This optimization problem yields to a clustering solution $\left\{\mathcal{B}_{i}^{\star}\right\}_{i=1}^{B}$ with low $\sum_{i=1}^{B} \sum_{m, n \in \mathcal{B}_{i}^{\star}}\left\|\mathbf{h}_{m}-\mathbf{h}_{n}\right\|^{2}$ and high $\sum_{p, q \notin \mathcal{B}_{i}}\left\|\mathbf{h}_{p}-\mathbf{h}_{q}\right\|^{2}$ for $i=1, \ldots, B$. 
In this context, the resulting matrix $\mathbf{G}^{H} \mathbf{G}$, being $\mathbf{G}$ constructed by the centroids of the clusters, will be likely a diagonally dominant matrix due to the clustering operation. Intuitively, this supports the precoding operation as it leverages the inter-cluster interference and promotes a low dissimilarity of the user-cluster channel vector constructed by the average of channel vectors of users belonging to the same cluster. In light of the above discussion, we claim that clustering users with similar channel vectors (in the Euclidean distance sense) might increase the resulting sum-rate. The shown numerical results evidence this notion.

Clearly, applying clustering techniques to heuristically and approximately solve the optimization problems in (3) and (4) would lead to different clustering solutions. In this paper, we tackle the combined problem in (1) by resorting to multigraph spectral clustering techniques.

\section{MULTIGRAPH SPECTRAL CLUSTERING}

In order to devise the existing clusters in a graph by means of spectral clustering, the main tool is the Laplacian matrix, which can be described as

$$
\mathbf{L}=\mathbf{S}-\mathbf{M}
$$

where $[\mathbf{S}]_{i j}=e^{\frac{-d_{i j}}{2 \nu^{2}}}$ is the similarity matrix for an arbitrary $\nu$ and $\mathbf{M}$ is the degree matrix defined as a diagonal matrix whose entries are the UTs degrees $m_{1}, \ldots, m_{K}$ such that $m_{i}=\sum_{j=1}^{K} s_{i j}$.

The value of $d_{i j}$ shall be the distance between the UTs. For the considered case, a relevant measurement of distance between UTs whenever precoding is going to be implemented is the Euclidean distance of the UT channel vectors: $d_{i j}^{\text {channel }}=$ $\left\|\mathbf{h}_{i}-\mathbf{h}_{j}\right\|^{2}$. In fact each cluster is represented by the centroid $\mathbf{g}_{i}$, which presents minimum quadratic distance to any of the channel vectors within a cluster. As stated above, in order to foster the multicast operation we can opt to group users considering their file requests. This is done by reconsidering the distance such that $d_{i j}^{\text {content }}=\left\|\mathbf{c}_{i}-\mathbf{c}_{j}\right\|^{2}$

In this Section we introduce two techniques able to deal with the optimization problem in (1). This clustering problem shall consider two views; namely, the UT channel vectors and the content demands. Thus, the techniques rely on both channel vector similarity matrix, $\mathbf{S}_{c}$, and the content request one, $\mathbf{S}_{f}$. Analogously, we refer to $\mathbf{L}_{c}$ and $\mathbf{L}_{f}$ as the Laplacian matrices of both the channel vectors feature and content request one.

We consider two different methods. While the first one relies on the combination of the different similarity matrices, the latter focuses on obtaining a consensus eigendecomposition of the different Laplacian matrices.

\subsection{Similarity Matrix Combination}

One alternative to consider multiple views of the same graph is to construct an equivalent similarity matrix that encompasses the characteristics of the different views. This technique is generally known as kernel composition and it has been successfully applied in many applications of kernelbased unsupervised learning [16].

In this paper we consider the next kernel compositions; namely, the kernel product $\mathbf{S}_{\text {prod }}=\mathbf{S}_{c} \circ \mathbf{S}_{f}$, where $\circ$ denotes the Hadamard product; and the kernel sum, $\mathbf{S}_{\text {sum }}=\mathbf{S}_{c}+\mathbf{S}_{f}$. Remarkably, despite its low computational complexity, the kernel sum is known to offer very good performances for certain problems such as text clustering [17]. On the other hand, the kernel product is equivalent to feature concatenation. That is, it is equivalent to perform the clustering with the vector $\mathbf{t}=\left(\mathbf{c}^{T}, \mathbf{h}^{T}\right)^{T}$ when considering the same $\nu$ for all views [18]. Once the equivalent similarity matrix is obtained, spectral clustering can be performed.

\subsection{Common Indication Matrix}

Another approach to clustering a multiview graph is the use of a regularized eigendecomposition [18]. Bearing in mind that the spectral clustering process is based on the eigendecomposition of the Laplacian matrix, we aim to construct a decomposition that simultaneously decomposes both $\mathbf{L}_{c}$ and $\mathbf{L}_{f}$. This can be done by optimizing

$$
\begin{aligned}
& \underset{\mathbf{V}_{c}, \mathbf{V}_{f}}{\operatorname{maximize}} \operatorname{Tr}\left(\mathbf{V}_{c}^{T} \mathbf{L}_{c} \mathbf{V}_{c}\right)+\operatorname{Tr}\left(\mathbf{V}_{f}^{T} \mathbf{L}_{f} \mathbf{V}_{f}\right) \\
& +\beta \operatorname{Tr}\left(\mathbf{V}_{c} \mathbf{V}_{c}^{T} \mathbf{V}_{f} \mathbf{V}_{f}^{T}\right) \\
& \text { subject to } \\
& \mathbf{V}_{c}^{T} \mathbf{V}_{c}=\mathbf{I}, \mathbf{V}_{f}^{T} \mathbf{V}_{f}=\mathbf{I} .
\end{aligned}
$$

As it can be observed, the term $\beta \operatorname{Tr}\left(\mathbf{V}_{c} \mathbf{V}_{c}^{T} \mathbf{V}_{f} \mathbf{V}_{f}^{T}\right)$ is employed to foster the similarity between $\mathbf{V}_{c}$ and $\mathbf{V}_{f}$. In other words, it promotes the clustering agreement among the files and the channel vectors domain. This method is coined as common indication matrix.

The optimization problem in (6) is a coupled non-convex problem which can be solved via an alternating optimization procedure. Once the alternating optimization has converged, the system designer has to choose to use either $\mathbf{V}_{f}$ or $\mathbf{V}_{c}$. No big differences are expected as the optimization problem closely related them.

Another interesting approach is to consider the optimization in (6) over a common solution [18]. This can be done by using a consensus variable as the non-convex optimization problem in (7). Again, the optimization problem is a non-convex coupled problem but an alternating optimization approach can provide relevant value solutions. Remarkably, both $\beta_{c}$ and $\beta_{f}$ can balance the different views and promote one view among other.This method is coined as common consensus indication matrix.

$$
\begin{aligned}
& \underset{\mathbf{V}_{c}, \mathbf{V}_{f}, \mathbf{V}_{a}}{\operatorname{maximize}} \operatorname{Tr}\left(\mathbf{V}_{c}^{T} \mathbf{L}_{c} \mathbf{V}_{c}\right)+\operatorname{Tr}\left(\mathbf{V}_{f}^{T} \mathbf{L}_{f} \mathbf{V}_{f}\right) \\
& +\beta_{c} \operatorname{Tr}\left(\mathbf{V}_{c} \mathbf{V}_{c}^{T} \mathbf{V}_{a} \mathbf{V}_{a}^{T}\right)+\beta_{f} \operatorname{Tr}\left(\mathbf{V}_{f} \mathbf{V}_{f}^{T} \mathbf{V}_{a} \mathbf{V}_{a}^{T}\right) \\
& \text { subject to } \\
& \mathbf{V}_{c}^{T} \mathbf{V}_{c}=\mathbf{I}, \mathbf{V}_{f}^{T} \mathbf{V}_{f}=\mathbf{I}, \mathbf{V}_{a}^{T} \mathbf{V}_{a}=\mathbf{I} .
\end{aligned}
$$




\section{NUMERICAL RESULTS}

We now demonstrate the benefits of our proposed method in multibeam satellite systems. For our simulations, we adopt the geostationary satellite channel model described in Section II. We consider $P=55$ Watts, $G_{R}=42.2 \mathrm{dBi}$ and $B_{W}=500$ MHz. The satellite operates at $20 \mathrm{GHz}$. The values of $a_{k n}$ have been obtained from a simulated real satellite array fed reflector. The $d_{k}$ values are also obtained for a certain satellite and UT location. All contents are assumed to have the same probability of request $(1 / C)$. All simulated values have been obtained considering 1000 Monte Carlo runs.
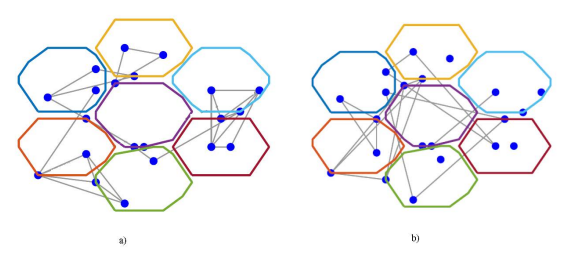

Fig. 1. One realization considering $K=21$ and $C=5$. Each beam is depicted by a different color and the vertex are plotted when the distance is larger than $10^{-4}$. In a) the graph of the channel vectors is presented while in b) it is shown the graph of content demands.

We first show two graph examples in Figure 1 considering both the channel vectors (a) and the content demands (b). The vertex connectivity is depicted whenever its weight is larger than $10^{-4}$. Along with the graph representation we have included the corresponding beampattern obtained as mentioned before. In this example we assumed $K=21$ and $C=5$. It can be observed that the channel vector graph tends to have a large connectivity between UTs that belong to close beams while the content does not follow this rule and it presents connection between UTs that are geographically separated. Clearly, both clustering techniques might lead to different solutions and; thus, our proposed multigraph approach results of great interest in this scenario.

To illustrate the performance of our proposed methods, Figure 2 shows the network efficiency (i.e. the objective function of the optimization problem in (1)) considering different values of $B$ for $N=10, K=300$ and $C=50$. The common indication matrix method has used a value of $\beta=0.5$. This value has shown the best performance for this setting.

The common indication matrix is the technique that yields the highest network efficiency for this setting. On the contrary, whenever the user clustering only considers the content requests (green line), it results to a very inefficient performance. It is important to remark that the similarity matrix sum technique, despite of its low complexity, offers a large weighted sum-rate. Whenever only $\mathbf{S}_{c}$ is considered, the network efficiency values are notably high.

As a benchmark, we also included the performance of $k$ means algorithm applied to the feature concatenation vector, $\mathbf{t}$, for each UT. As it is depicted in Figure 2, the performance of this method is substantially lower compared to the other techniques based on spectral clustering.

We now evaluate the performance of the common consen-

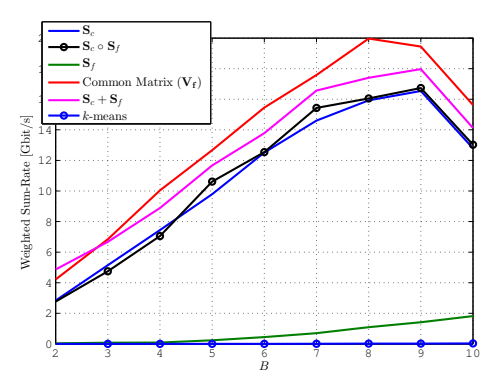

Fig. 2. Multigraph spectral clustering for different number of groups and $300 \mathrm{UTs}$ with $C=50$ contents.

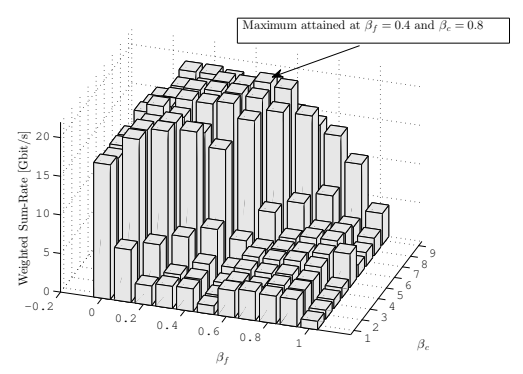

Fig. 3. Performance of consensus common matrix indicator $\left(\mathbf{V}_{a}\right)$ for $0 \leq \beta_{f} \leq 1$ and $0 \leq \beta_{c} \leq 1$. It has been considered 300 UTs with $C=50$ contents.

sus indication matrix technique of the optimization problem in (7) for different values of $\beta_{f}$ and $\beta_{c}$ when considering the same setting as before and with $B=8$. We can observe in Figure 3, high values of $\beta_{f}$ (i.e. the consensus solution is closer to the only-content clustering), the network efficiency performance decreases severely. In other words, the relations of the UTs channel vectors are more relevant than the content ones for this particular example. The optimal tuple is $\beta_{f}=0.4$ and $\beta_{c}=0.8$ which leads to a reduced performance gain with respect to the common matrix indicator of the optimization problem in (6) (i.e. a $1 \%$ by comparing the results for $B=8$ in Figure 2 and the optimal tuple weighted sumrate in Figure 3) gain. This is indicated by an arrow in Figure 3.

\section{CONCLUDING REMARKS}

We presented two algorithms to group users in a multibeam content delivery satellite network with multicast precoding. To do so, we have used the multigraph spectral clustering framework that resulted to properly model the different connections of the problem. The two proposed techniques behaved well in the synthetic data examples. In light of the results, it might be interesting to study other multigraph clustering capabilities such as the case of UTs with different detection capabilities (e.g. single user detection or multiple user detection). This can be useful for non-orthogonal multiple access applications. 


\section{REFERENCES}

[1] N. Wang, N. Nouwell, C. Ge, B. Evans, Y. Rahulan, M. Boutin, J. Desmauts, K. Liolis, C. Politis, S. Votts, and G. Poziopoulou, "Satellite Support for Enhanced Mobile Broadband Content Delivery in 5G," in 2018 IEEE International Symposium on Broadband Multimedia Systems and Broadcasting (BMSB), June 2018, pp. 1-6.

[2] M. A. Vazquez, A. Perez-Neira, D. Christopoulos, S. Chatzinotas, B. Ottersten, P. D. Arapoglou, A. Ginesi, and G. Taricco, "Precoding in Multibeam Satellite Communications: Present and Future Challenges," IEEE Wireless Communications, vol. 23, no. 6, pp. 88-95, December 2016.

[3] K. U. Storek and A. Knopp, "Fair User Grouping for Multibeam Satellites with MU-MIMO Precoding," in GLOBECOM 2017 - 2017 IEEE Global Communications Conference, Dec 2017, pp. 1-7.

[4] N. Zorba, M. Realp, and A. I. Perez-Neira, "An improved partial CSIT random beamforming for multibeam satellite systems," in 2008 10th International Workshop on Signal Processing for Space Communications, Oct 2008, pp. 1-8.

[5] G. Taricco, "Linear Precoding Methods for Multi-Beam Broadband Satellite Systems," in European Wireless 2014; 20th European Wireless Conference, May 2014, pp. 1-6.

[6] D. Christopoulos, S. Chatzinotas, and B. Ottersten, "Multicast Multigroup Precoding and User Scheduling for Frame-Based Satellite Communications," IEEE Transactions on Wireless Communications, vol. 14, no. 9, pp. 4695-4707, Sept 2015.

[7] V. Joroughi, M. Á. Vázquez, and A. I. Pérez-Neira, "Generalized Multicast Multibeam Precoding for Satellite Communications," IEEE Transactions on Wireless Communications, vol. 16, no. 2, pp. 952-966, Feb 2017.

[8] Alessandro Guidotti, Alessandro Vanelli-Coralli, Giorgio Taricco, and Guido Montorsi, "User Clustering for Multicast Precoding in Multi-Beam Satellite Systems," arXiv preprint arXiv:1706.09482, 2017.

[9] Alessandro Guidotti and Alessandro Vanelli-Coralli, "Geographical Scheduling for Multicast Precoding in Multi-Beam Satellite Systems," arXiv preprint arXiv:1804.06614, 2018.
[10] M. Á. Vázquez, M. R. B. Shankar, C. Kourogiorgas, P. D. Arapoglou, V. Icolari, S. Chatzinotas, A. D. Panagopoulos, and A. I. Pérez-Neira, "Precoding, Scheduling and Link Adaptation in Mobile Interactive Multibeam Satellite Systems," IEEE Journal on Selected Areas in Communications, pp. 1-1, 2018.

[11] Geoffrey H Ball and David J Hall, "A clustering technique for summarizing multivariate data," Systems Research and Behavioral Science, vol. 12, no. 2, pp. 153-155, 1967.

[12] Ulrike Luxburg, “A Tutorial on Spectral Clustering," Statistics and Computing, vol. 17, no. 4, pp. 395-416, Dec. 2007.

[13] M. Á. Vázquez and A. I. Pérez-Neira, "Spectral Clustering for Beam-Free Satellite Communications," in IEEE Global Conference on Signal and Information Processing (GlobalSIP 2018), November 2018, Anaheim (USA), Nov. 2018.

[14] S. Bickel and T. Scheffer, "Multi-view clustering," in Fourth IEEE International Conference on Data Mining (ICDM'04), Nov 2004, pp. 19-26.

[15] Christopher M Bishop, Pattern recognition and machine learning, Springer, 2006.

[16] Thorsten Joachims, Nello Cristianini, and John Shawe-Taylor, "Composite Kernels for Hypertext Categorisation," in Proceedings of the Eighteenth International Conference on Machine Learning, San Francisco, CA, USA, 2001, ICML '01, pp. 250-257, Morgan Kaufmann Publishers Inc.

[17] Corinna Cortes, Mehryar Mohri, and Afshin Rostamizadeh, "Learning Non-Linear Combinations of Kernels," in $A d$ vances in Neural Information Processing Systems 22, Y. Bengio, D. Schuurmans, J. D. Lafferty, C. K. I. Williams, and A. Culotta, Eds., pp. 396-404. Curran Associates, Inc., 2009.

[18] Abhishek Kumar, Piyush Rai, and Hal Daume, "Coregularized Multi-view Spectral Clustering," in Advances in Neural Information Processing Systems 24, J. Shawe-Taylor, R. S. Zemel, P. L. Bartlett, F. Pereira, and K. Q. Weinberger, Eds., pp. 1413-1421. Curran Associates, Inc., 2011. 